\title{
Temperature Effect on Dynamic Properties of Magnetorheological Elastomers
}

\author{
Yan-Xiang WAN ${ }^{1, a}$, Ye-Ping XIONG ${ }^{1, b}$ and Shen-Ming ZHANG ${ }^{2, c}$ \\ ${ }^{1}$ University of Southampton, UK \\ 2Lloyd's Register, UK \\ ayw8g12@soton.ac.uk, bY.Xiong@soton.ac.uk, cShengming.Zhang@Ir.org \\ *Yanxiang Wan
}

\begin{abstract}
Keywords: Magnetorheological elastomer, dynamic mechanical analysis, temperature, viscoelasic modeling
\end{abstract}

\begin{abstract}
Magnetorheological elastomers (MREs) are typically comprised of magnetic particles and non-magnetic matrix. Their mechanical properties are dependent on many factors, among which temperature is very important yet lack of understanding due to limited studies. In this paper, experimental study has been carried out to characterise the dynamic properties of MREs under different temperature and magnetic fields. By using the obtained experimental data, the viscoelastic model for rheological properties of MREs is presented based on the generalized Maxwell model and fractional Maxwell model. The experimental results show that temperature has an important influence on the dynamic modulus of MREs made from silicone rubber embedded with iron particles subjected to uniaxial compression. It is found that there is a transition behavior of MREs at about $50^{\circ} \mathrm{C}$ for dynamic modulus, i.e. below which the storage modulus of MRE is first decreases with the increasing of temperature up to $50^{\circ} \mathrm{C}$ and then increases with the temperature increased.
\end{abstract}

\section{Introduction}

Magnetorheological Elastomer (MRE) is a group of new smart composite material based on its unique elastic and rheological properties that can be changed continuously, rapidly and reversibly responding to the applied magnetic field. MRE's real-time controllable stiffness and damping properties have many advantages and offer wide potential applications to vibration control in various engineering fields, attracting much more attention in recent years. Typically, MRE consists of magnetic particle filler and a non-magnetic matrix with some additives. Their mechanical properties are dependent on many factors such as microstructure, volume fraction of particles, magnetic field, temperature, and excitation amplitude and frequency.

It was found that the stiffness of anisotropic MREs seemed to be more sensitive to magnetic field and thus the MR effect for anisotropic MREs was slightly larger than isotropic ones in most cases [1, 2]. The stiffness of MREs increased as the intensity of the applied magnetic field increased [3, 4] and the maximum MR effect can be achieved when the iron particle volume fraction was about $30 \%[5,6]$. The MR effect decreased obviously with the increment of strain amplitude and the maximum value of MR effect can be obtained at relatively small strain amplitudes when the strongest interaction between the particles occurred [7].

MRE based devices are often exposed to a wide temperature range. MRE exhibits a temperature dependent softening and stiffening ability at low and high temperature respectively, which can have an adverse effect on MRE based devices. Zhang et al [8] and Ju et al [9] carried out the experimental investigation respectively on the temperature dependent magneto-mechanical properties of MRE samples by using carbonyl iron particles dispersed in different rubbery matrixes: polyurethane (PU) rubber [9] and mixed rubber matrices (cis-polybutadiene rubber and natural rubber) [8]. The dynamic mechanical properties of MRE samples were tested by a rheometer and the influence of temperature and magnetic field on the storage modulus and loss modulus were evaluated. 
Once the dependent properties of MREs are determined by using dynamic mechanical analysis (DMA) tests, a viscoelastic constitutive model is employed in finite element analysis (FEA) in order to design and assess the performance of the MRE based devices for semi-active vibration control. There are several viscoelastic models available to describe the rheological behaviors of viscoelastic materials as a function of temperature and frequency. Among these models, the Maxwell model and Kelvin-Voigt model are two basic phenomenological models that consist of connecting the spring and dashpot in serial and parallel respectively. For MRE materials, the linear viscoelastic models can be used to characterize the rheological behavior when the applied strain is in small range $[4,10]$. In order to obtain a good fit of DMA experiment data and accurate results by using FEA, these phenomenological models are modified based on the fractional derivative model $[11,12]$.

In this paper, the anisotropic MRE samples are designed, manufactured, and tested. The influence of temperature and magnetic field on the rheological properties of MREs is obtained and discussed. The linear viscoelastic models are presented based on the generalized Maxwell model and the fractional Maxwell model to describe the dynamic modulus, including storage modulus and loss modulus. The model parameters are identified by using the experiment data of MRE samples.

\section{Experimental}

\section{Experiment Method}

The MRE samples in this paper were composed of 30\% iron particles and $70 \%$ silicon rubber in volume fraction mixed and cured under room temperature. The iron particles were purchased from Sigma-Aldrich company ( $\geq 99.5 \%$, grain size 5-9 $\mu \mathrm{m})$. The silicone rubber was purchased from Wacker Chemie AG (Germany). According to the instructions from the company for the silicon rubber, Elastosil A was mixed with Elastosil B as 10:1 in volume fraction and then the iron particles were added. The mixture was blended by stirrer to make sure the iron particles were well distributed in the matrix. Finally, the mixed materials were placed in the aluminum moulds and left to cure for more than 20 hours. In additional, for the anisotropic MRE samples the moulds were placed between two permanent magnets producing a stable magnetic field of about $300 \mathrm{mT}$. The magnetic field, during curing and testing, was generated by using cylindrical grade N42 neodymium permanent magnets (purchased from E-magnets UK) with a diameter of $40 \mathrm{~mm}$ and thickness of $5 \mathrm{~mm}$. The MRE samples of cylinder column $13 \mathrm{~mm}$ height by $28 \mathrm{~mm}$ radius were prepared according to the BS ISO 4664-1:2011.
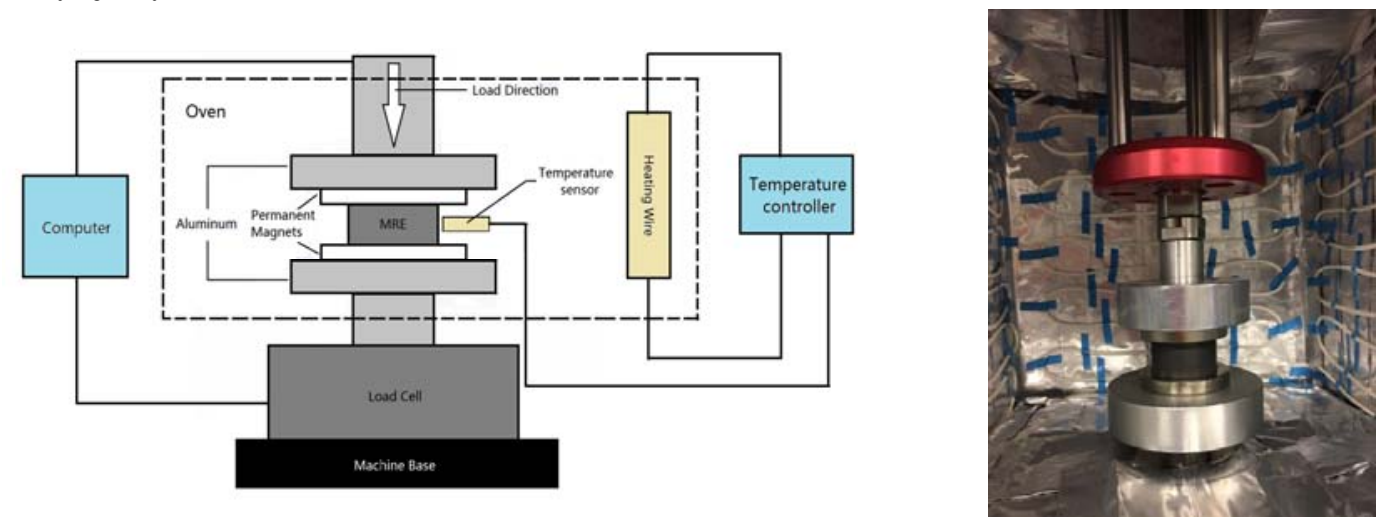

Fig. 1 Experiment setup for DMA test of MRE sample under uniaxial compression

The DMA tests of the MRE samples were performed by using Instron test machine (E1000 Electro plus) and self-manufactured oven in the Transport Systems Research Laboratory. As shown in Fig. 1, the aluminum silicate layer was placed between the shell and the aluminum foil to guarantee the specimen temperature stay around the desired temperature. Heating wire was well arranged inside the oven. A temperature control panel was applied to control the heating system and make sure the inside temperature stay within desired range. The specimen temperature was monitored by a thermos 
detector. The magnetic field was generated by a pair of cylindrical grade N42 neodymium permanent magnets and the strain amplitude and the excitation frequency were controlled through the displacement channel and the resulting force was measured by the force channel.

\section{Results and Discussion}

The DMA tests of the MRE samples were performed under uniaxial compression with a pre-strain of $5 \%$. Strain amplitude was $1 \%$, excitation frequencies were from 0 to $60 \mathrm{~Hz}$, magnetic field intensity were 0 and $500 \mathrm{mT}$, and test temperature was from room temperature (RT) to $60^{\circ} \mathrm{C}$. All the MRE samples were preloaded and unloaded for ten cycles before the DMA test to avoid the Mullins effect. Each set of tests was carried out with three pairs of samples independently and each test was repeated twice for each pair of samples, the experimental data are averaged to present the curve or surface to guarantee the reliability of the data.

The dynamic mechanical properties of MRE samples under uniaxial compression were determined. The influence of temperature and magnetic field on the dynamic properties of MRE were obtained and discussed. Fig. 2 shows the dependent hysteresis loops of the MRE samples under uniaxial compression.

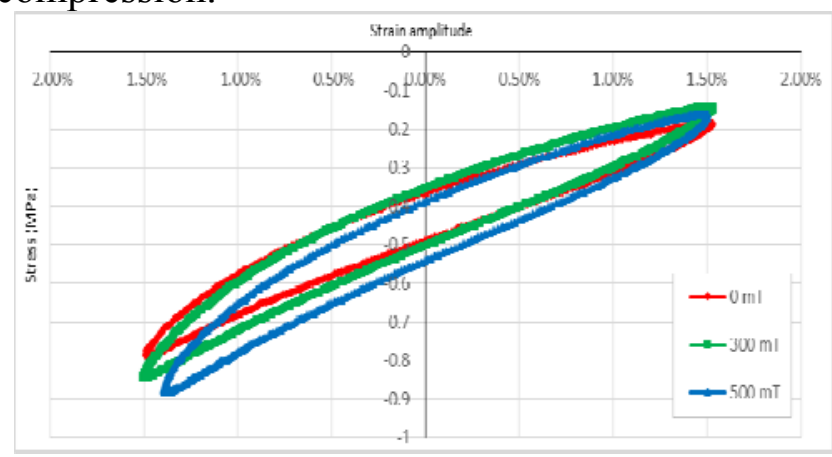

(a)

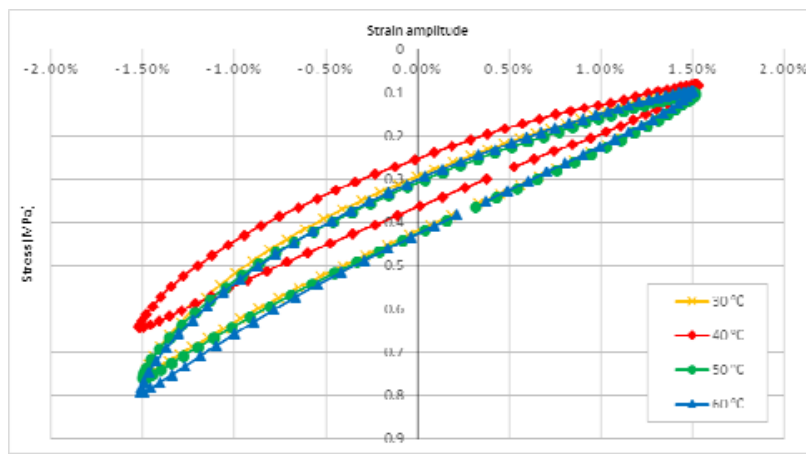

(b)

Fig. 2 Dependence of hysteresis loops on: (a) magnetic field, and (b) temperature
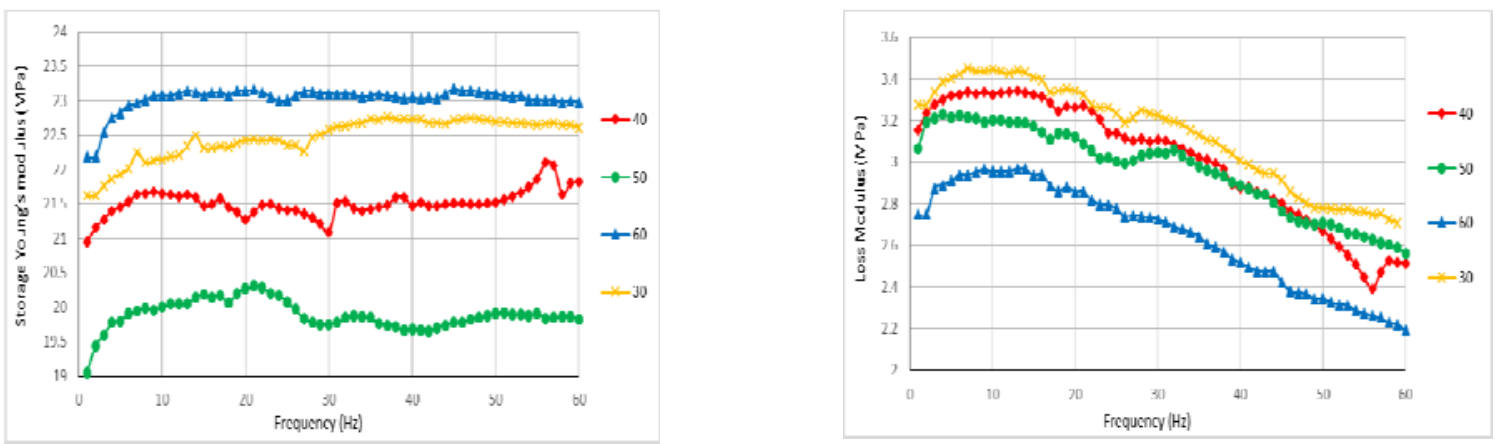

(a)
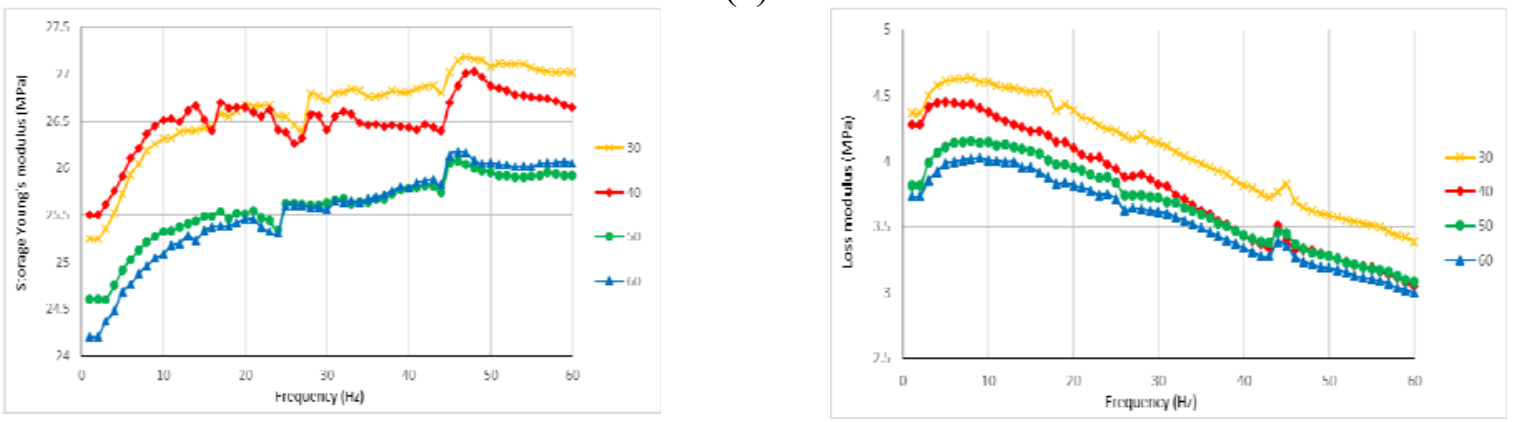

(b)

Fig. 3 Dependence of storage modulus and loss modules on frequency and temperature in: (a) non-magnetic field, and (b) magnetic field of $500 \mathrm{mT}$ 
It can be observed from Fig. 2(a) that the MRE samples obviously became stiffer with the increase of magnetic field intensity. Based on the hysteresis loops, the complex modulus can be obtained from the slope of the line from the minimum strain point to the maximum strain point. The loss angle can be calculated from the area within the hysteresis loop and the storage and loss moduli can be obtained as well. Fig. 2(b) shows the temperature dependent hysteresis loops of the MRE samples. Large differences in the hysteresis loop shape and position can be observed between different temperatures. Compared with the other impact factors above, the temperature had bigger influence on hysteresis loop of MRE materials.

Fig. 3 shows the dependent dynamic modulus of the MRE samples with different magnetic fields and temperatures. The experimental results revealed that the storage modulus initially decreases with temperature, reached the minimum value at $50^{\circ} \mathrm{C}$ and then started to increase with further increasing of temperature. The change of the material storage modulus with temperature can be mainly divided into two stages by $50^{\circ} \mathrm{C}$ temperature point. At the first stage, the storage modulus of MRE material usually decreased with the increasing of temperature, but in stage two it became the opposite, the storage modulus started to increase with the increment of temperature. The loss modulus decreased with the increasing of temperature and the decrement of the curves were more remarkable when the MRE samples were under both magnetic field and higher temperature.

\section{Viscoelastic modeling}

\section{Maxwell-type Models}

The generalized Maxwell model is comprised of a spring, several Maxwell elements, and a dashpot in parallel as shown in Figure 4(a), the corresponding complex modulus is equal to the sum of the complex modulus of a spring, a dashpot, and each Maxwell element.

$$
E^{*}(i \omega)=E_{0}+\sum_{k=1}^{m} \frac{E_{k} \omega^{2} \tau_{k}^{2}}{1+\omega^{2} \tau_{k}^{2}}+i\left(\eta_{0} \omega+\sum_{k=1}^{m} \frac{E_{k} \omega \tau_{k}}{1+\omega^{2} \tau_{k}^{2}}\right)
$$

where $E_{0}$ is relaxed modulus of the spring, $\eta_{0}$ is viscosity of the dashpot, $\mathrm{m}$ is the number of Maxwell elements, $E_{k}, \eta_{k}$, and $\tau_{k}=\eta_{k} / E_{k}$ denotes the relaxation strength, viscosity, and relaxation time of the $\mathrm{k}^{\text {th }}$ Maxwell element respectively.

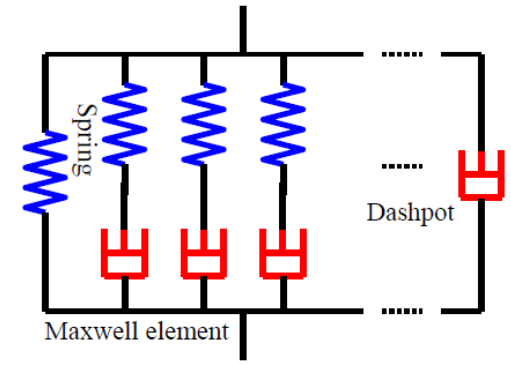

(a)

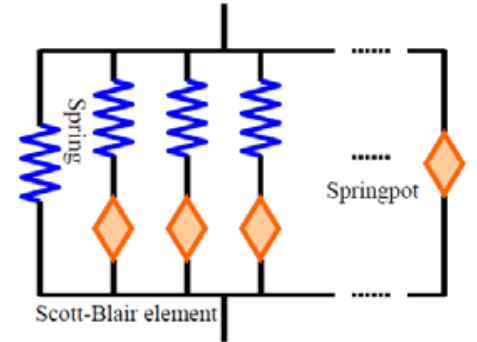

(b)

Figure 4 Linear viscoelastic models: (a) the generalized Maxwell model, and (b) the fractional Maxwell model

In order to improve the generalized Maxwell model to describe the elastic and viscous behavior of viscoelastic material based on the experimental data, the Scott-Blair element (also called fractional Maxwell element) was introduced in which the dashpot was replaced with a springpot as shown in Figure 4(b). The corresponding complex modulus is equal to the sum of the complex modulus of a spring, a springpot, and each Scott-Blair element. 


$$
\begin{aligned}
E^{*}(i \omega) & =E_{0}+\left(\eta_{0} \omega\right)^{\alpha} \cos \frac{\alpha \pi}{2}+\sum_{k=1}^{m} E_{k} \frac{\left(\omega \tau_{k}\right)^{2 \alpha}+\left(\omega \tau_{k}\right) \cos \frac{\alpha \pi}{2}}{1+\left(\omega \tau_{k}\right)^{2 \alpha}+2\left(\omega \tau_{k}\right)^{\alpha} \cos \frac{\alpha \pi}{2}} \\
& +i\left[\left(\eta_{0} \omega\right)^{\alpha} \sin \frac{\alpha \pi}{2}+\sum_{k=1}^{m} \frac{E_{k}\left(\omega \tau_{k}\right)^{\alpha} \sin \frac{\alpha \pi}{2}}{1+\left(\omega \tau_{k}\right)^{2 \alpha}+2\left(\omega \tau_{k}\right)^{\alpha} \cos \frac{\alpha \pi}{2}}\right]
\end{aligned}
$$

\section{Parameter Identification}

The model parameters $\left(E_{k}, \tau_{k}, k=0,1,2, \ldots, \mathrm{m}\right)$ in the generalized Maxwell model, or $\alpha$ and $\left(E_{k}, \tau_{k}\right.$, $k=0,1,2, \ldots, \mathrm{m})$ in the fractional Maxwell model can be identified respectively based on experimental data of the MRE samples. The number of Maxwell element, $\mathrm{m}$, is determined in order to obtain a good fit of the experimental data of MRE samples. In this paper, the model parameters are identified by using a least square method and optimization algorithm to minimizing the difference between the experimental data and model predicted results of the magnitude of the complex modulus.

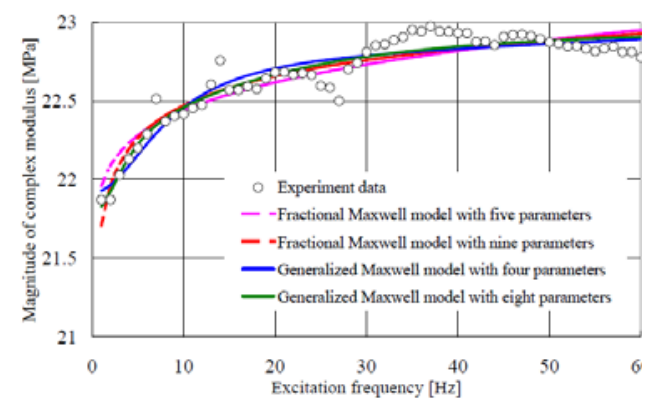

(a)

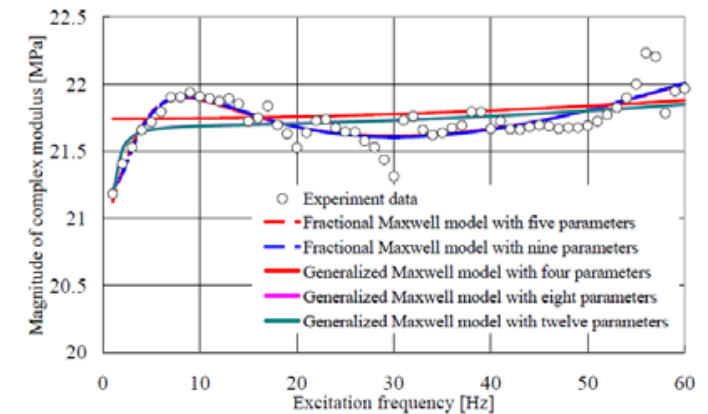

(b)

Fig. 5 Comparison between experimental data and prediction results of the magnitude of complex modulus of the MRE samples in non-magnetic field and at temperature of: (a) $30^{\circ} \mathrm{C}$, and (b) $40^{\circ} \mathrm{C}$

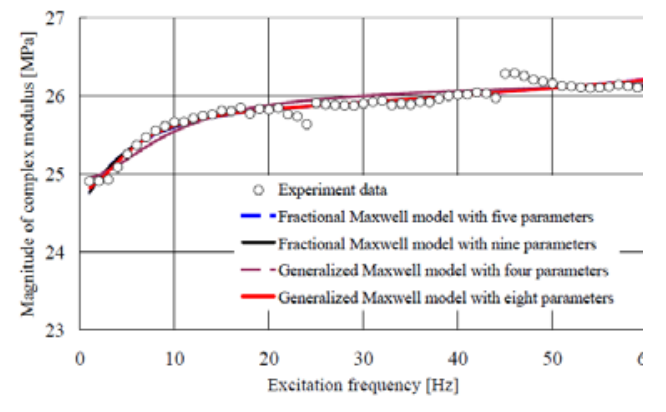

(a)

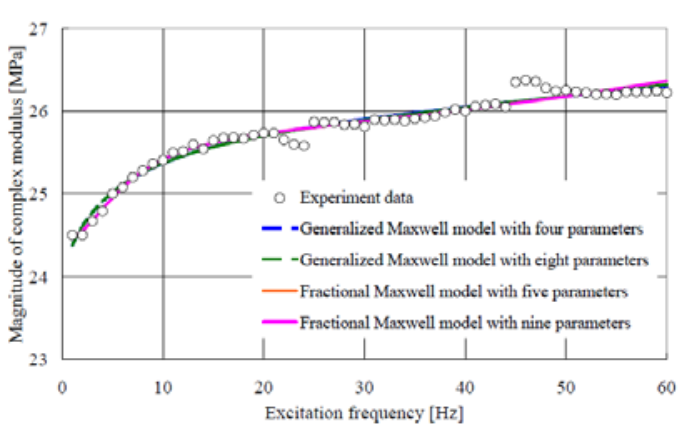

(b)

Fig. 6 Comparison between experimental data and prediction results of the magnitude of complex modulus of the MRE samples under magnetic field of $500 \mathrm{mT}$ and temperature of: (a) $50^{\circ} \mathrm{C}$, and (b) $60^{\circ} \mathrm{C}$

Fig. 5 and Fig. 6 present the magnitude of the complex modulus obtained by the MRE sample experiments and the viscoelastic model predictions by using the generalized Maxwell model and fractional Maxwell model. The difference exists between the experimental data and model prediction results. The fractional Maxwell model gives a better fitting result than the generalized Maxwell model and the improvement of fitting result is not obvious when increasing the number of model parameters. 


\section{Conclusion}

In this paper, the anisotropic MRE samples based on silicone rubber embedded with iron particles are designed, manufactured, and tested. The influence of temperature and magnetic field on the dynamic mechanical properties of the MRE samples is determined. The experimental results show that temperature has an important impact on the dynamic modulus of MRE samples based on silicone rubber embedded with iron particles under uniaxial compression. The loss modulus decreases with the increasing of temperature while the storage modulus initially decreases with the increasing of temperature, reaches the minimum value at $50^{\circ} \mathrm{C}$ and then starts to increase with further increasing temperature.

The linear viscoelastic models are presented based on the generalized Maxwell model and fractional Maxwell model to describe the storage modulus and loss modulus. The model parameters are identified by minimizing the difference between the experimental data and model predictions of MRE samples. The fractional Maxwell model gives a better fitting result than the generalized Maxwell model and the improvement of fitting result is not obvious when increasing the number of model parameters.

\section{Reference}

[1] H. Böse and R. Röder, Magnetorheological elastomers with high variability of their mechanical properties, J. Phys. Conf. Ser. 149 (2009) 012090.

[2] J. Kaleta, M. Królewicz, D. Lewandowski, Magnetomechanical properties of anisotropic and isotropic magnetorheological composites with thermoplastic elastomer matrices, Smart Mater. Struct. 20 (2011) 085006.

[3] H.J. Jung, S.J. Lee, D.D. Jang, I.H. Kim, J.H. Koo, F. Khan, Dynamic characterization of magneto-rheological elastomers in shear mode, IEEE Trans. Magn. 45 (2009) 3930-3933.

[4] W.H. Li, Y. Zhou, T.F. Tian, Viscoelastic properties of MR elastomers under harmonic loading, Rheol Acta, 49 (2010) 733-740.

[5] M. Lokander and B. Stenberg, Improving the magnetorheological effect in isotropic magnetorheological rubber materials, Polymer Testing, 22 (2003) 677-680.

[6] S.H. Eem, H.J. Jung, J.H. Koo, Seismic performance evaluation of an MR elastomer-based smart base isolation system using real-time hybrid simulation, Smart Mater. Struct. 22 (2013) 055003.

[7] R. Li and L.Z. Sun, Dynamic mechanical behavior of magnetorheological nanocomposites filled with carbon nanotubes, Appl. Phys. Lett. 99 (2011) 131912.

[8] W. Zhang, X.L. Gong, S.H. Xuan, W.Q. Jiang, Temperature-dependent mechanical properties and model of magnetorheological elastomers, Ind. Eng. Chem. Res. 50 (2011) 6704-6712.

[9] B.X. Ju, T. Tang, D.Y. Zhang, B.L. Yang, M. Yu, C.R. Liao, Temperature-dependent dynamic mechanical properties of magnetorheological elastomers under magnetic field, J. Magn. Magn. Mater. 374 (2015) 283-288.

[10] M. Sasso, G. Palmieri, G. Chiappini, D. Amodio, Frequency dependent mechanical behaviour elastomers under uniaxial cyclic loading, Proc. SEM Ann. Conf. New Mexico USA 2009.

[11] J. Kaleta, D. Lewandowski, G. Zietek, Inelastic properties of magnetorheological composites: II. Model, identification of parameters, Smart Mater. Struct. 16 (2007) 1954-1960.

[12] M. Norouzi, S.M.S.A. Alehashem, H. Vatandoost, Y.Q. Ni, M.M. Shahmardan, A new approach for modeling of magnetorheological elastomers, J. Intell. Mater. Syst. Struct. 27 (2015) 1121-1135. 